\title{
Stratégies d'adaptation des organismes subventionnaires en sciences humaines et sociales au Canada et au Québec aux compressions budgétaires gouvernementales
}

\section{MATHIEU ALBERT* \\ Université du Québec à Montréal}

\section{RÉSUMÉ}

La présente étude vise à évaluer dans quelle mesure deux aspects de la stratégie déployée par les organismes subventionnaires (OS) canadiens et québécois en sciences humaines et sociales (SHS) pour faire face aux compressions budgétaires gouvernementales ont atteint leurs objectifs. Plus spécifiquement, nous avons vérifié si la mise sur pied et la consolidation des programmes de recherche orientée au début des années 90 ont permis aux OS en SHS de générer des revenus additionnels à ceux octroyés par les gouvernements, et comparer ces revenus à ceux générés en sciences naturelles et génie (SNG) dans le cadre de programmes équivalents. Nous avons également vérifié dans quelle mesure les professeurs en SHS ont transformé leurs pratiques de recherche afin de répondre favorablement à l'accroissement de l'offre de subventions de recherche orientée. Les résultats suggèrent que les programmes de recherche orientée n'ont pas véritablement générés de nouveaux revenus en SHS et, qu'en fait, ces programmes ont eu pour effet d'accentuer les inégalités économiques entre les SHS et les SNG.

\footnotetext{
* L'auteur tient à remercier Robert Dalpé, professeur au département de science politique de l'Université de Montréal et chercheur au CIRST, pour ses commentaires et suggestions qui ont contribué à raffiner l'analyse de la présente étude.
} 
Pour ce qui est des professeurs, ceux-ci n'ont pas véritablement modifié leur pratiques de recherche dans le but de répondre à l'offre croissante de subventions de recherche orientée.

\section{ABSTRACT}

This study sought to evaluate to what degree the strategies employed by Canadian and Quebecois funding agencies in the social sciences and humanities were successful in dealing with government spending restrictions. More specifically, it examined whether the oriented research programs that were established and consolidated at the beginning of the 1990s have enabled funding agencies in the social sciences and humanities to supplement government funding, and it compared this revenue to that which was generated within equivalent programs in the natural sciences and engineering. It also examined to what extent professors in the social sciences and humanities have transformed their research practices to meet the increased number of grants available for oriented research. The results suggest that oriented research programs have not generated new revenues in the social sciences and humanities and that these programs have in fact amplified the existing financial inequalities between the social and natural sciences. The results also seem to indicate that professors did not in effect modify their research practices to adapt to the growing number of grants offered for oriented research.

\section{INTRODUCTION}

Bien que la demande de pertinence sociale et économique de la recherche adressée par les gouvernements canadien et québécois aux organismes subventionnaires (OS) ne constitue pas un phénomène nouveau (Chapman \& Farina, 1983; Dalpé \& Anderson, 1995; Dalpé \& Gingras, 1990; Gingras, Godin \& Trépanier, 1999), celle-ci s'affirme aujourd'hui avec une intensité accrue. Le resserrement des finances publiques incite, en effet, les OS à recourir à des sources de financement autres que celles issues des crédits parlementaires et à accroître la part des fonds provenant des acteurs économiques, politiques et sociaux (v.g. les entreprises, ministères, organismes sans but lucratif, associations, 
groupes communautaires et politiques). Parmi les initiatives mises en oeuvre à cette fin, la création et/ou la consolidation de programmes de subvention de recherche orientée, ainsi que le renouvellement du discours visant à convaincre les gouvernements et les éventuels pourvoyeurs de fonds de la rentabilité socio-économique de la recherche universitaire semblent avoir occupé une place prédominante.

En ce qui a trait au discours, reprenant certains thèmes de la conception du monde "économiciste» caractéristique des champs politique et économique actuels (Bourque \& Beauchemin, 1994; Beauchemin, Bourque \& Duchastel, 1995), les OS ont fait valoir l'importance du soutien de l'activité scientifique universitaire en raison de son rôle-clé dans l'intensification de la compétitivité nationale au sein du nouvel ordre économique mondial. L'extrait qui suit illustre la stratégie discursive utilisée par les OS pour mettre en valeur la rentabilité de l'activité scientifique en sciences humaines et sociales:

[La recherche en sciences humaines] apporte des informations sur des structures organisationnelles innovatrices... et des moyens de favoriser à la fois la motivation et la productivité; elle permet en outre de connaître les cultures, les langues et les traditions de nos partenaires commerciaux... Ce sont là des éléments que le Canada ne saurait négliger s'il veut demeurer concurrentiel sur les marchés internationaux. La recherche en sciences humaines nous donne les outils dont nous avons besoin pour prévoir les grands changements structurels de l'économie... (CRSH, 1990a, pp. 1-2)'

Les appels lancés aux chercheurs universitaires par les responsables des OS afin qu'ils mettent en valeur plus activement les retombées socio-économiques de leurs travaux auprès des décideurs politiques et économiques semblent également avoir été plus nombreux et plus insistants au cours de la dernière décennie. L'extrait suivant constitue un exemple éloquent de cet appel en faveur d'une diffusion accrue des retombées de l'activité scientifique universitaire auprès de la société:

De nombreux intérêts publics se disputent les crédits fédéraux, qui sont en baisse. Nous devons présenter au gouvernement des arguments très solides pour qu'il continue 
d'appuyer la recherche universitaire, et le Conseil a besoin d'aide de la communauté de recherche pour faire valoir les meilleurs arguments en la faveur du maintien voire de l'accroissement de nos efforts de recherche et de formation de chercheurs... Nous devons expliquer en termes concrets ce que nous faisons et pourquoi. Les arguments abstraits portant sur "l'importance de la recherche" sont beaucoup moins convaincants que des exemples qui illustrent comment nos activités contribuent à la vie quotidienne et répondent aux préoccupations des Canadiens. (CRSNG, 1994a, pp. 1-2) ${ }^{2}$

Dans la présente étude, nous nous concentrons sur les initiatives touchant la mise sur pied et/ou la consolidation des programmes de recherche orientée depuis le début des années 90 , et ce, pour les OS oeuvrant dans le domaine des sciences humaines et sociales (SHS). L'objectif consiste à examiner la réponse des deux catégories d'acteurs concernés par le développement des programmes de subvention de recherche orientée, soit les pourvoyeurs de fonds (i.e., les partenaires) d'une part, et les professeurs-chercheurs en SHS d'autre part. Nous visons à évaluer, dans un premier temps, dans quelle mesure les programmes de recherche orientée ont permis aux OS en SHS de générer des revenus additionnels à ceux octroyés par les gouvernements entre 89-90 et 96-97, et de comparer ces revenus à ceux générés par les programmes de recherche orientée des OS en sciences naturelles et génie (SNG). Au plan de l'analyse des dynamiques sociales, ce premier volet servira à documenter la problématique largement discutée depuis quelques années, portant sur les effets sur la recherche universitaire de l'insertion progressive du mode d'allocation des ressources dans la logique du marché.

En ce qui a trait au second volet de l'étude, touchant aux pratiques des professeurs-chercheurs en SHS, nous visons à vérifier dans quelle mesure ces derniers ont répondu favorablement à la croissance de l'offre de subventions de recherche orientée et voir s'ils ont intensifié leurs activités de recherche visant à répondre à la demande sociale de connaissances. Ce second volet permettra d'évaluer dans quelle mesure les analyses de Gibbons et al. (1994), selon lesquelles il y aurait eu une 
progression de la production de connaissances en lien avec les divers contextes d'application se vérifient au plan de la demande de subventions en SHS au Québec et au Canada.

\section{L'état des connaissances sur les organismes subventionnaires dans un contexte de compression budgétaire au Québec et au Canada}

Si la transformation des politiques scientifiques et technologiques des gouvernements canadien et québécois et leur impact sur les activités des chercheurs est bien documentée (cf., entre autres, Davis, 1993; Dufour \& Gingras, 1993; Gingras, Godin \& Trépanier, 1999; Limoges, 1990; Trépanier, 1992), les effets de l'intensification du recours au partenariat comme mode de financement de la recherche sur le partage des ressources entre les secteurs de connaissance demeurent moins bien connus. Deux études réalisées au début des années 90 (Leclerc, 1991; Leclerc \& Gingras, 1993) ont montré que la participation accrue de l'industrie au financement de la recherche universitaire avait eu pour effet d'accentuer les inégalités économiques entre les secteurs de connaissance.

Dans le cadre d'une étude portant sur les activités de collaboration des chercheurs universitaires avec l'industrie, les partenaires institutionnels (ministères, hôpitaux, organismes sans but lucratif, etc.) et les autres chercheurs universitaires, Godin et Landry (1995) et Godin (1998) ont fait ressortir que les chercheurs en génie et en sciences biomédicales collaborent davantage avec l'industrie que leurs collègues en SHS, ces derniers privilégiant surtout des liens de collaboration avec les partenaires institutionnels. Bien que les travaux de Godin et Landry et de Godin ne visaient pas à cerner l'impact de l'intensification du recours au partenariat sur les ressources disponibles pour les OS et les divers secteurs de connaissance, leurs résultats, à l'instar de ceux de Leclerc et Gingras, mettent en relief le caractère fortement différencié des relations qu'entretiennent les disciplines avec les différentes catégories de partenaires.

En ce qui a trait aux efforts déployés par les gouvernements et les OS afin d'orienter les activités des chercheurs, Chapman et Farina (1983) et Dalpé et Gingras (1990) ont mis en lumière la difficulté pour ceux-ci de concrétiser leur ambition à cet égard. Chapman et Farina ont 
montré que les programmes stratégiques mis sur pied en 1977 par le Conseil de recherches en sciences naturelles et génie (CRSNG) n'avaient pas réussi à modifier l'agenda des problèmes de recherche des scientifiques. Ces derniers se seraient plutôt servis de ces programmes comme source additionnelle de financement pour des recherches déjà menées et financées ailleurs. Dans leur étude sur l'impact du programme stratégique en énergie solaire du CRSNG et du ministère de l'Énergie, des mines et des Ressources du Canada, Dalpé et Gingras (1990) sont parvenus à des conclusions similaires. Les tentatives du gouvernement fédéral pour orienter les activités des chercheurs n'auraient obtenu qu'un succès relatif; certains chercheurs ayant bénéficié de subventions stratégiques auraient continué à travailler dans des domaines qui n'étaient qu'indirectement liés aux thématiques ciblées par le programme. Dans la même optique, une étude réalisée par le CRSH en 1996 (SSHRC, 1996) mettait en relief l'impact limité exercé par les modifications apportées au programme de Subventions stratégiques sur les pratiques des professeurs-chercheurs en SHS. Alors que le CRSH souhaitait, lors de la relance du programme en 1990, que les chercheurs établissent des partenariats avec divers organismes (CRSH, 1990b), l'étude révèle qu'après cinq ans, seulement $56 \%$ des projets présentés comportaient un partenaire - un taux qui était toutefois en croissance, depuis $34 \%$ en 1991. De plus, parmi les demandes où les professeurs s'étaient adjoints la participation d'un partenaire, il s'agissait dans $29 \%$ des cas d'un partenaire fantôme, celui-ci n'ayant, soit, pas été informé par les chercheurs que son nom était utilisé dans le cadre d'une demande de subvention, soit, peu ou pas informé de l'état d'avancement des travaux lorsque le projet avait été accepté (SSHRC, 1996).

Bien que la faible capacité d'intervention des instances publiques sur le développement de la recherche universitaire paraît être un fait reconnu, deux études récentes (Gingras, Godin \& Trépanier, 1999; Godin \& Trépanier, 1995) montrent que le gouvernement fédéral a néanmoins accru son influence sur celle-ci au cours des années 90 . Ce dernier a en effet instauré de nouveaux mécanismes d'attribution des subventions dans certains programmes du CRSNG et dans les Réseaux de centre d'excellence permettant aux industries d'exprimer leurs 
besoins en matière de Recherche et Developpement. Alors que l'évaluation par les pairs avait constitué jusque-là le mode exclusif d'attribution des subventions, favorisant ainsi le maintien de l'autonomie relative du champ scientifique face aux demandes des champs politique et économique (Bourdieu, 1975, 1991, 1992; Pollack, 1975), les mécanismes mis en place par le gouvernement fédéral autorisent désormais les acteurs industriels à prendre part à l'élaboration des critères d'évaluation ainsi qu'au processus de sélection des projets. Cette ouverture au monde industriel a pour effet d'importer au sein du champ scientifique des critères d'évaluation qui, jusque-là, lui étaient étrangers, telle l'applicabilité des résultats et la pertinence économique des travaux.

\section{MÉTHODOLOGIE}

Pour connaître la contribution respective des trois catégories de pourvoyeurs de fonds ciblées par notre enquête au financement de la recherche universitaire en SHS, nous avons examiné la contribution annuelle des entreprises, des organismes sans but lucratif (OSBL) et des organismes publics et parapublics (OPP) à la recherche au cours de la période 89-90 à 96-97 dans les programmes Initiatives conjointes du CRSH et Actions concertées du Fonds FCAR et du CQRS. Ces programmes de subvention constituent les trois principaux programmes par lesquels les OS en SHS ont accès à des sources de revenus autres que celles provenant des crédits parlementaires. Les Initiatives conjointes du CRSH ont été lancées en 89-90; les Actions concertées du CQRS, en 1993. Les Actions concertées du Fonds FCAR ont été créées en 76-77, mais ont été «redynamisées» au début des années 90 afin d'accroître la part du financement des partenaires (Fonds FCAR, 1993, p. 17, 30, 51; 1994, p. 7). Ces trois programmes de subvention se caractérisent par le fait que le sujet de la recherche est défini par les partenaires et que ces derniers sont autorisés à participer à l'évaluation et à la sélection des projets soumis par les chercheurs. ${ }^{3}$

Pour comparer le niveau de financement des partenaires en SHS avec celui en SNG, nous avons utilisé les données compilées par le 
CRSNG par année et par catégorie de partenaires. Ces données nous ont été transmises par l'organisme.

Pour être en mesure d'estimer le niveau de financement annuel pour chacune des trois catégories de partenaire - les entreprises, les OSBL et les OPP - et de faire ressortir les tendances en SHS, nous avons dû, dans certains cas, et dans des proportions variables, ventiler les données des quatre OS en SHS.

Pour ce qui est du Fonds FCAR, étant donné que l'organisme a pour mandat de financer des travaux tant en SHS qu'en SNG, nous avons désagrégé les données disponibles et les avons complétées par des informations recueillies directement auprès de l'OS. ${ }^{4}$

Pour ce qui est du volet de l'enquête portant sur l'impact de la création et/ou de la consolidation des programmes de subvention de recherche orientée sur les pratiques des professeurs-chercheurs en SHS, nous avons comparé l'évolution de la demande de subventions dans les programmes de recherche orientée à l'évolution de la demande dans les programmes de recherche non orientée. ${ }^{5}$

Dans ce second volet, aux trois programmes de subvention de recherche déjà cités (Initiatives conjointes, Actions concertées/Fonds FCAR et Actions concertées/CQRS), nous avons ajouté à notre corpus l'ensemble des autres programmes de subventions de recherche orientée en SHS administrés par les OS ciblés par notre enquête. Au CRSH, il s'agit du programme de Subventions stratégiques; un programme créé en 1978 sous le nom de Subventions thématiques, mais révisé en 1990 suite aux recommandations du rapport Paquet visant à susciter un intérêt accru de la part des universitaires à l'endroit de la recherche orientée et à accentuer la pertinence sociale des thèmes proposés (SSHRC, 1989; 1996). Au CQRS, il s'agit des Programmes de subventions à la recherche (à l'exception des subventions d'aide au transfert ou à la diffusion des résultats, qui ne sont pas des subventions de recherche mais de transfert de connaissances), des Programmes de subventions d'équipe et des Programmes de bourses de carrière (à l'exception des bourses de doctorat et de postdoctorat qui sont destinées aux étudiants). Le Fonds FCAR ne comptent aucun programme de subvention de recherche orientée autre que celui des Actions concertées. 
Ce second ensemble de programmes se distingue des trois programmes de recherche orientée cités plus haut en ce sens que la thématique de recherche est définie par l'organisme et non par des partenaires. Certains de ces programmes exigent des chercheurs qu'ils s'associent à des partenaires avant de soumettre une demande, alors que d'autres, bien qu'ils encouragent le partenariat, n'en font pas une condition d'admissibilité.

L'ensemble des programmes de subventions de recherche non orientée en opération au cours de la période ciblée a servi de point de comparaison à l'évolution de la demande de subventions dans les programmes orientés. Au CRSH, les programmes de subvention de recherche non orientée sont: les Subventions ordinaires de recherche, les Grandes subventions de recherche et les Grands travaux de recherche concertée. Au Fonds FCAR, les programmes sont: Soutien aux équipes de recherche, Soutien à l'établissement de nouveaux chercheurs et Centres de recherche. Le CQRS ne compte aucun programme de subvention de recherche non orientée puisque la totalité de ses programmes s'inscrit dans la Politique de la santé et du bien-être du gouvernement du Québec telle qu'établie en 1992 par le ministère de la Santé et des Services sociaux du Québec. Les chercheurs ne peuvent donc y soumettre des projets élaborés hors de toutes contraintes thématiques. Les projets doivent nécessairement s'inscrire dans les objectifs socio-communautaires du CQRS. ${ }^{6}$

Pour ce qui est des données provenant du Fonds FCAR, en raison du fait que les programmes de subvention de cet organisme s'adressent à la fois aux chercheurs en SHS et en SNG, nous avons dû compléter les données compilées par l'organisme (Fonds FCAR, 1996) par des informations obtenues auprès des responsables des programmes.

Soulignons enfin que notre étude n'examine pas l'impact sur la recherche de la progression des contrats de recherche directement établis entre les entreprises, les OSBL, les OPP et les professeurs-chercheurs. ${ }^{7}$ Notre étude se concentre plutôt sur l'impact du partenariat tel qu'il se manifeste dans la recherche subventionnée par les OS. 


\section{Évolution des fonds octroyés aux organismes subventionnaires par les gouvernements du Canada et du Québec depuis la fin des années 80}

Avant d'examiner les stratégies d'adaptation déployées par les OS pour faire face aux compressions budgétaires gouvernementales, il convient de faire le portrait de l'évolution des fonds publics qui leur ont été octroyés depuis la fin des années 80 .

Comme l'illustrent les graphiques 1 et 2 , le ralentissement de la croissance puis la diminution des crédits gouvernementaux canadien et québécois au chapitre de la recherche universitaire s'est accompagnée de fluctuations historiques relativement importantes au cours des dernières années, et ce, tant dans le domaine des SNG, que dans ceux des sciences biomédicales et des SHS. Ces fluctuations budgétaires présentent des courbes à peu près identiques au CRSNG, au Conseil de recherches médicales (CRM), au CRSH et au Fonds pour la formation de chercheurs et l'aide à la recherche (Fonds FCAR). Ainsi, après avoir connu une période de croissance jusqu'au début des années 90 , le budget de ces OS s'est stabilisé pendant quelques années avant de décroître à partir du milieu des années 90 .

Les crédits gouvernementaux destinés au CRSNG ont été réduits de $12 \%$ entre $92-93$ et $97-98$, passant de 499 millions $\$$ à 440 millions $\$$ (en $\$$ courants); ceux du CRM ont subi une réduction de $10 \%$ entre 94-95 et 97-98, passant de 265 millions $\$$ à 238 millions $\$$ ( $\$$ courants); et ceux du CRSH, une réduction de $11 \%$ entre $92-93$ et $96-97$, passant de 102 millions $\$$ à 91 millions \$ (\$ courants). En 98-99, ces trois OS ont toutefois vu leurs crédits parlementaires rétablis au niveau de 94-95 par le gouvernement fédéral, récupérant ainsi une partie de ce qu'ils avaient perdu au cours des années précédentes. Convertie en dollars constants de 1986, cette augmentation n'a toutefois ramené leur budget respectif qu'au niveau où il était en 88-89 pour le CRSNG et le CRSH et 89-90 pour le CRM.

Le gouvernement du Québec a réduit pour sa part de $4 \%$ sa contribution au Fonds FCAR entre $94-95$ et 98-99, faisant passer son budget de 52 millions $\$$ à 50 millions $\$$ (\$ courants). Le Fonds de 


\section{Graphique 1}

Crédits parlementaires alloués par le gouvernement fédéral au CRSNG, au CRM et au CRSH de 87-88 à 98-99 en dollars courants et en dollars constants de 1986

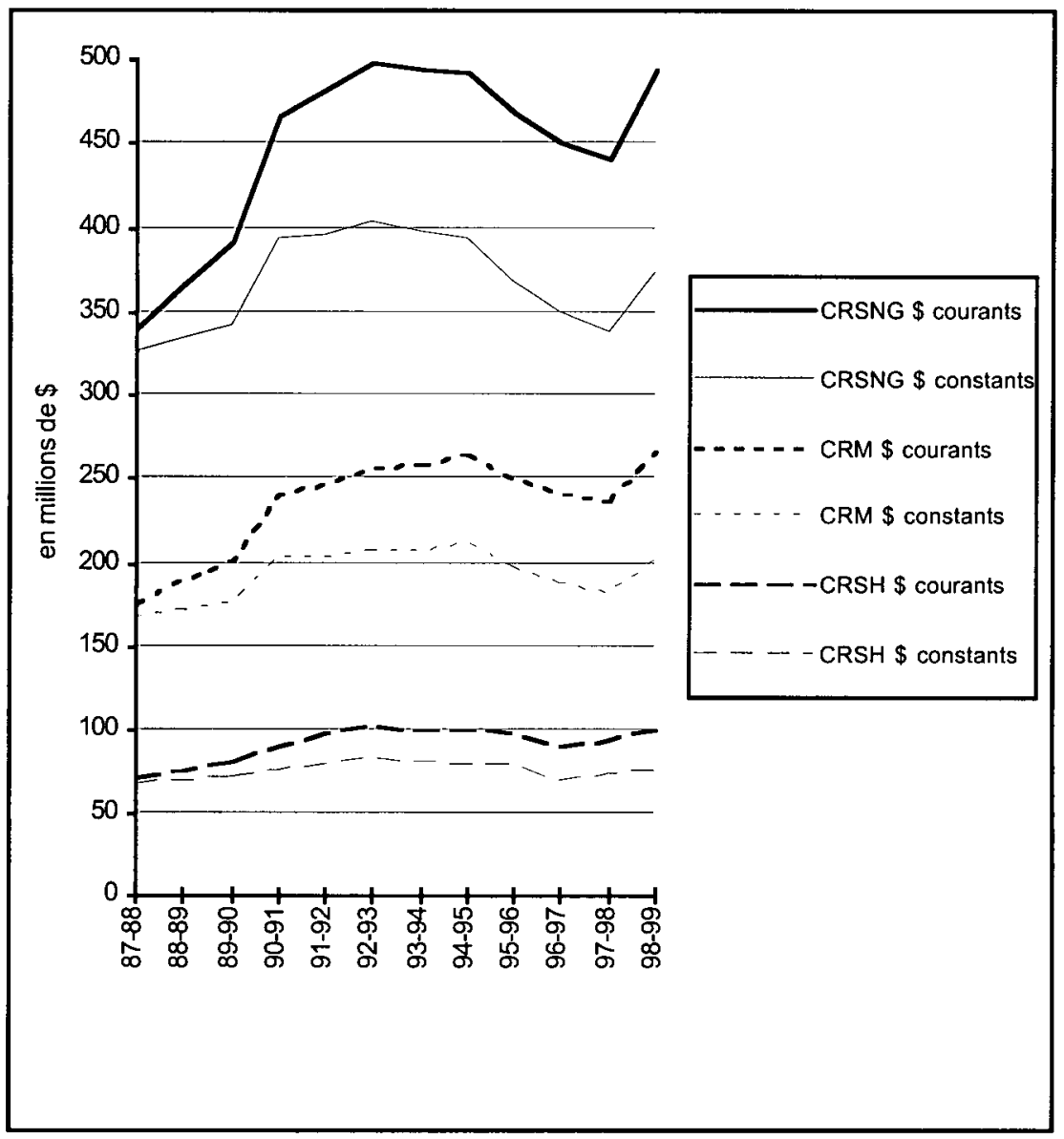

Sources: Rapports annuels des OS. Les montants incluent les crédits parlementaires pour les subventions à la recherche, les bourses et les frais de fonctionnement. 


\section{Graphique 2}

Crédits parlementaires alloués par le gouvernement du Québec au Fonds FCAR, au FRSQ et au CQRS de 87-88 à 98-99 en dollars courants et en dollars constants de 1986

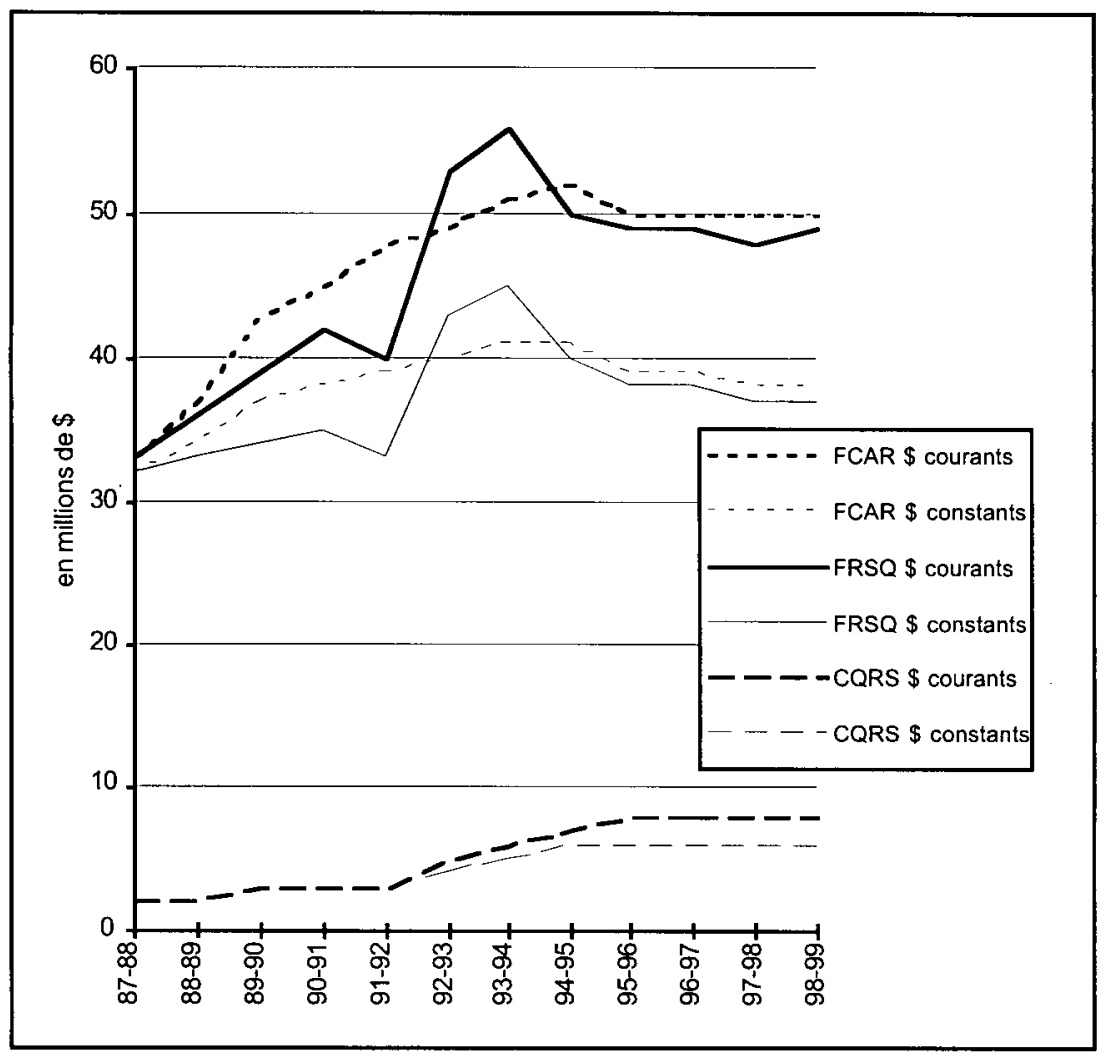

Sources: Rapports annuels des OS. Les montants incluent les crédits parlementaires pour les subventions à la recherche, les bourses et les frais de fonctionnement. 
recherche en santé du Québec (FRSQ) a vu pour sa part son budget réduit de $14 \%$ entre $93-94$ et $97-98$, passant de 56 millions $\$$ à 48 millions $\$$ ( $\$$ courants).

Contrairement à l'ensemble des OS, le budget du Conseil québécois de la recherche sociale (CQRS) a connu, pour sa part, une forte augmentation relative, de plus de $321 \%$, au cours de la période $87-88$ à 96-97, passant de 1,9 millions $\$$ à 8 millions $\$$ ( $\$$ courants). Cette augmentation peut s'expliquer par le fait que la totalité des programmes de subvention du CQRS sont des programmes de subvention de recherche orientée. La mission «sociale» de l'organisme coïnciderait avec l'intensification de l'orientation instrumentale de l'appui à la recherche souhaitée par les intervenants des milieux politique et social.

\section{La réponse des pourvoyeurs de fonds aux besoins de financement des organismes subventionnaires en sciences humaines et sociales}

Examinons en premier lieu la réponse des pourvoyeurs de fonds aux besoins de financement des OS en SHS. La comparaison des données apparaissant aux graphiques 3 et 4 fait voir que la réponse des pourvoyeurs de fonds varie considérablement selon qu'il s'agit du domaine des SHS ou des SNG. Alors que le secteur des SHS (Graphique 3) semble susciter peu d'intérêt chez les partenaires, tel que mesuré à l'aune des sommes investies, le secteur des SNG (Graphique 4) semble bénéficier d'un appui à la fois constant et croissant de la part de ces derniers. La comparaison des données suggère que l'appui à la recherche universitaire provenant des entreprises, des OSBL et des OPP est incomparablement plus substantiel dans le domaine des SNG que dans celui des SHS. Alors que la contribution totale des trois catégories de pourvoyeurs de fonds au secteur des SNG s'élevait à près de 35 millions \$ en 89-90 (en dollars courants), leur contribution au secteur des SHS ne totalisait que 312000 \$. En 96-97, la contribution des partenaires au secteur des SNG se chiffrait à plus de 70 millions $\$$ alors qu'elle ne dépassait pas 2 millions \$ en SHS.

Bien que les fonds octroyés aux SHS affichent une croissance relative, en pourcentage, cinq fois plus élevée que ceux octroyés aux SNG (525\% pour les SHS contre $100 \%$ pour les SNG), les sommes 
Graphique 3

Contribution des trois catégories de partenaires (entreprises, OSBL, OPP) aux programmes de partenariat en SHS du CQRS, du CRSH et du Fonds FCAR (secteur SHS) de 89-90 à 96-97

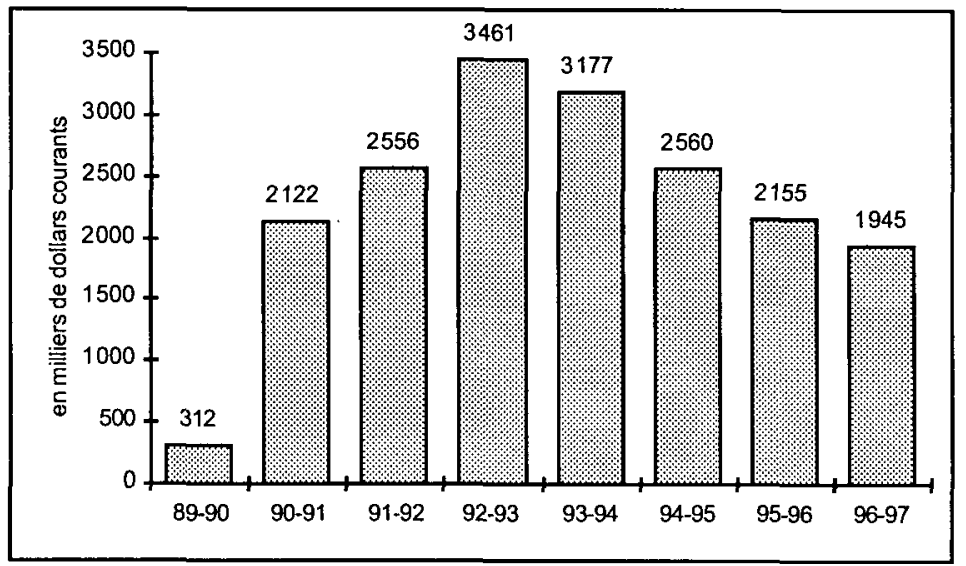

\section{Graphique 4}

Contribution des trois catégories de partenaires (entreprises, OSBL, OPP) aux programmes de partenariat en SNG du CRSNG et du Fonds FCAR (secteur SNG) de 89-90 à 96-97

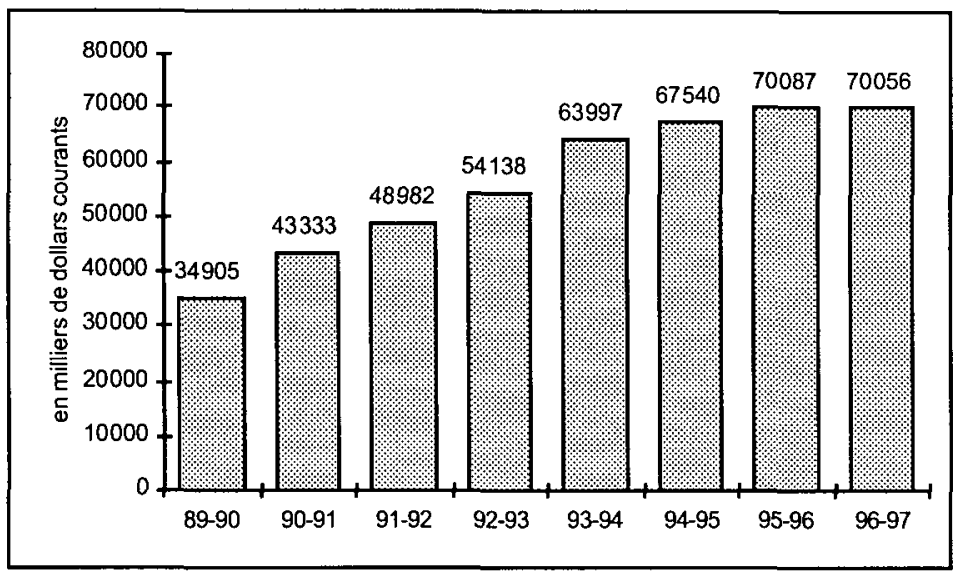

The Canadian Journal of Higher Education

Volume XXX, No. 3, 2000 
investies en SHS demeurent à ce point minimes qu'elles sont insuffisantes pour leur permettre d'atteindre un niveau de financement proportionnellement comparable à celui des SNG. Par exemple, pour la seule année 96-97, les professeurs-chercheurs en SNG bénéficiaient de ressources additionnelles aux crédits gouvernementaux 35 fois supérieures à celles auxquelles avaient accès les professeurs en SHS. Bien qu'il soit reconnu que les besoins financiers liés à l'activité scientifique en SHS sont inférieurs à ceux en SNG, on peut toutefois douter que ces besoins s'établissent à un niveau 35 fois inférieur à ceux des SNG. À cet égard, le taux de succès obtenu par les demandeurs de subvention en SHS et en SNG constitue un indicateur de l'écart entre le niveau de satisfaction des besoins financiers dans les deux secteurs disciplinaires. L'examen des données des OS révèlent en effet que le taux de succès dans le programme de Subventions de recherche du CRSNG atteignait 76\% en 1996-1997 pour un total de plus de 2777 demandes (CRSNG, 1999), alors que ce taux ne s'établissait qu'à 30\% pour l'ensemble des programmes de recherche en SHS au CRSH, au Fonds FCAR (secteur SHS) et au CQRS, pour un total de 2452 demandes (Albert, 1999). Au-delà du fait que les coûts de la recherche en SHS soient inférieurs à ceux en SNG, ces données suggèrent que les ressources disponibles en SHS sont, toute proportion gardée, largement moins abondantes qu'en SNG.

$\mathrm{Si}$ on compare maintenant la proportion de l'appui financier des pourvoyeurs de fonds à l'appui reçu des gouvernements (Graphique 5), on observe que l'intensification du recours au partenariat comme mode de financement de la recherche universitaire a eu pour effet de canaliser vers les SNG des ressources en croissance plus rapide que celles canalisées vers les SHS. En effet, alors que la proportion de la contribution des partenaires aux SNG par rapport aux crédits gouvernementaux enregistrait une augmentation de $76 \%$ entre $89-90$ et 96-97, passant de $8 \%$ à $15 \%$ des crédits gouvernementaux, la proportion du financement des partenaires en SHS demeurait à peu près inchangée, passant de seulement $0,31 \%$ en $89-90$ à $1,6 \%$ en 96-97. Le graphique 5 montre également que la proportion de la contribution des partenaires en SNG est en progression constante, attestant de l'implication croissante de 
Graphique 5

Proportion des fonds alloués par les partenaires aux secteurs des SHS et des SNG de 89-90 à 96-97 en comparaison des crédits versés par les gouvernements du Québec et du Canada

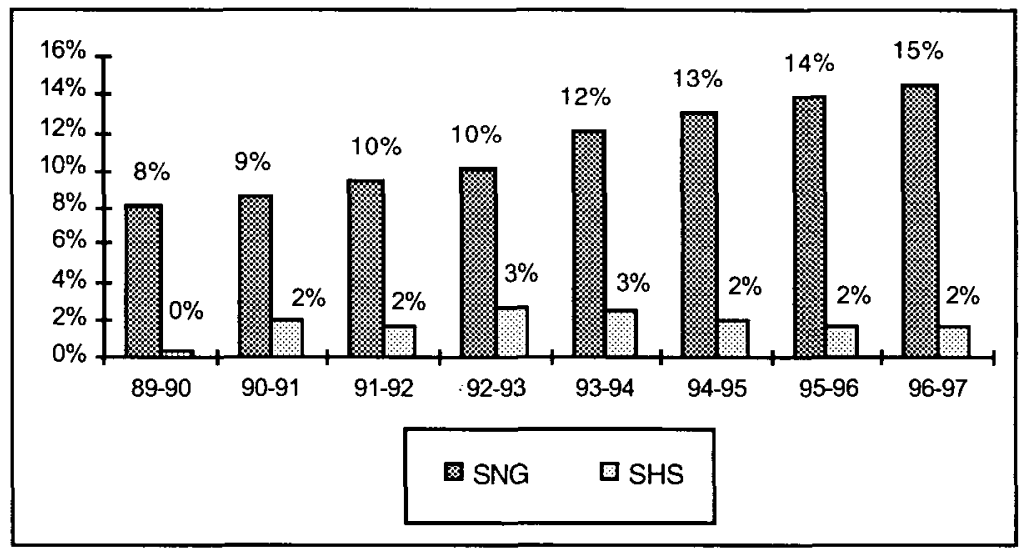

ces derniers dans les activités de recherche en SNG. En comparaison, les partenaires des SHS se montrent beaucoup plus réservés. Après avoir enregistré une légère progression au cours de la première moitié de la période ciblée - la proportion de leurs investissements atteignant 3\% des crédits gouvernementaux en $92-93$ et 93-94 - cette proportion décline par la suite pour se stabiliser autour de 2\% au cours des années 94-95 à 96-97. Il importe de souligner toutefois que, bien que la participation des partenaires au domaine des SNG soit en croissance, les montants qu'ils allouent demeurent inférieurs à ceux versés par les gouvernements; ces derniers demeurant les principaux pourvoyeurs de fonds de la recherche universitaire (Godin \& Trépanier, 1995).

Nos résultats suggèrent que le recours au partenariat par les OS en vue de diversifier leurs sources de revenu a donné lieu à une accentuation de l'écart entre les ressources disponibles en SNG et en SHS. Deux facteurs peuvent contribuer à expliquer cet état de fait. Le premier tient à l'importance de plus en plus grande qu'occupe le potentiel de valorisation commerciale des résultats dans les choix relatifs 
au financement de la recherche, le second, aux mesures fiscales adoptées par les gouvernements pour favoriser les investissements en R-D.

En ce qui a trait au premier facteur, il semble plausible d'avancer que la place grandissante faite aux partenaires - qui sont majoritairement constituées par les entreprises (Graphique 7) - dans le financement de la recherche favorise l'essor d'un mode d'allocation des ressources où le critère des retombées économiques et sociales occupe une place croissante. Le pouvoir d'attraction qu'exercent les SNG auprès des pourvoyeurs de fonds pourrait ainsi s'expliquer par leur capacité de générer des résultats éventuellement convertibles en innovations techniques ou en produits commercialisables. En revanche, l'intérêt mitigé suscité par les SHS s'expliquerait par leur incapacité de générer des résultats dotés d'un pouvoir comparable. Le potentiel de rentabilité des secteurs scientifiques et, par extension, des OS eux-mêmes, aurait ainsi tendance à s'affirmer de plus en plus comme l'un des éléments prépondérants du principe d'allocation des ressources.

Nos résultats convergent avec ceux de Leclerc (1991), de Leclerc et Gingras (1993), et de Slaughter et Leslie (1997). Quoique compilées sur la base d'une classification disciplinaire différente de la nôtre, les données analysées par Leclerc (1991) et Leclerc et Gingras (1993) mettent en lumière que les secteurs dont les résultats présentent un potentiel de commercialisation (les sciences de la santé et les sciences appliquées, cf., Leclerc \& Gingras, 1993) réussissent à canaliser un volume de ressources beaucoup plus important que les secteurs dépourvus d'un potentiel comparable (les sciences pures et les sciences sociales, cf., Leclerc \& Gingras, 1993). Les résultats de leur étude font ressortir également que les entreprises et les institutions du secteur privé financent prioritairement les secteurs disciplinaires dotés d'une capacité de valorisation commerciale élevée, au détriment des secteurs ne détenant pas une capacité équivalente.

L'enquête de Slaughter et Leslie (1997) montre, pour sa part, que les compressions budgétaires gouvernementales ont conduit les universités de quatre pays industrialisées à intensifier leur recours à des sources de financement alternatives, les obligeant de plus en plus à adopter des stratégies de gestion sous-tendues par les exigences du marché. Au plan 
disciplinaire, les auteurs soulignent que certains secteurs de connaissance, telles les «technosciences», auraient tiré avantage de l'insertion des activités universitaires dans la dynamique de l'économie de marché, alors que d'autres secteurs, notamment en sciences humaines et sociales (v.g. la philosophie, l'étude des langues, les Lettres, les sciences de l'éducation, les sciences religieuses), auraient enregistré une décroissance des ressources disponibles (Slaughter \& Leslie, 1997). Bien que le découpage disciplinaire de Slaughter et Leslie ne soit pas identique au nôtre, les résultats de leur étude convergent avec nos analyses: l'intensification du recours au partenariat en tant que source de financement de la recherche universitaire aurait entraîné un déclin relatif de la marge de manoeuvre des SHS, alors qu'elle aurait accrue la part relative de celle des $\mathrm{SNG}$ (ou, à tout le moins, des secteurs les plus appliqués des SNG).

Pour ce qui est du second facteur pouvant expliquer la disparité entre les ressources des partenaires canalisées vers les SHS et les SNG, celuici tient aux mesures fiscales adoptées par les gouvernements pour favoriser l'investissement en R-D. Bien qu'elles n'établissent pas une distinction nette entre les secteurs des SNG et des SHS, ces mesures sont accompagnées par des règles d'admissibilité ayant pour effet de favoriser les investissements en SNG et en sciences biomédicales (SBM) et non en SHS. ${ }^{8}$

Il convient de noter, par ailleurs, que cette politique d'exemption fiscale fait en sorte qu'une part importante des investissements issus du secteur privé est en fait défrayée par les fonds publics (Dalpé, 1999; Leclerc \& Gingras, 1993); ce qui relativise l'apport du secteur privé.

\section{La contribution des partenaires, par catégorie, au financement de la recherche}

Examinons maintenant sur une base individuelle la part allouée par chacune des trois catégories de pourvoyeurs de fonds au financement des SHS et des SNG. Si on se concentre tout d'abord sur les SHS (Graphique 6), on constate que les OPP constituent presque la source unique de financement alternative aux crédits gouvernementaux à laquelle les SHS ont accès. Les données font ressortir, en effet, que les 
Graphique 6

Contribution des partenaires, par catégorie, aux programmes de partenariat en sciences sociales du Fonds FCAR, du CQRS et du CRSH de 89-90 à 96-97

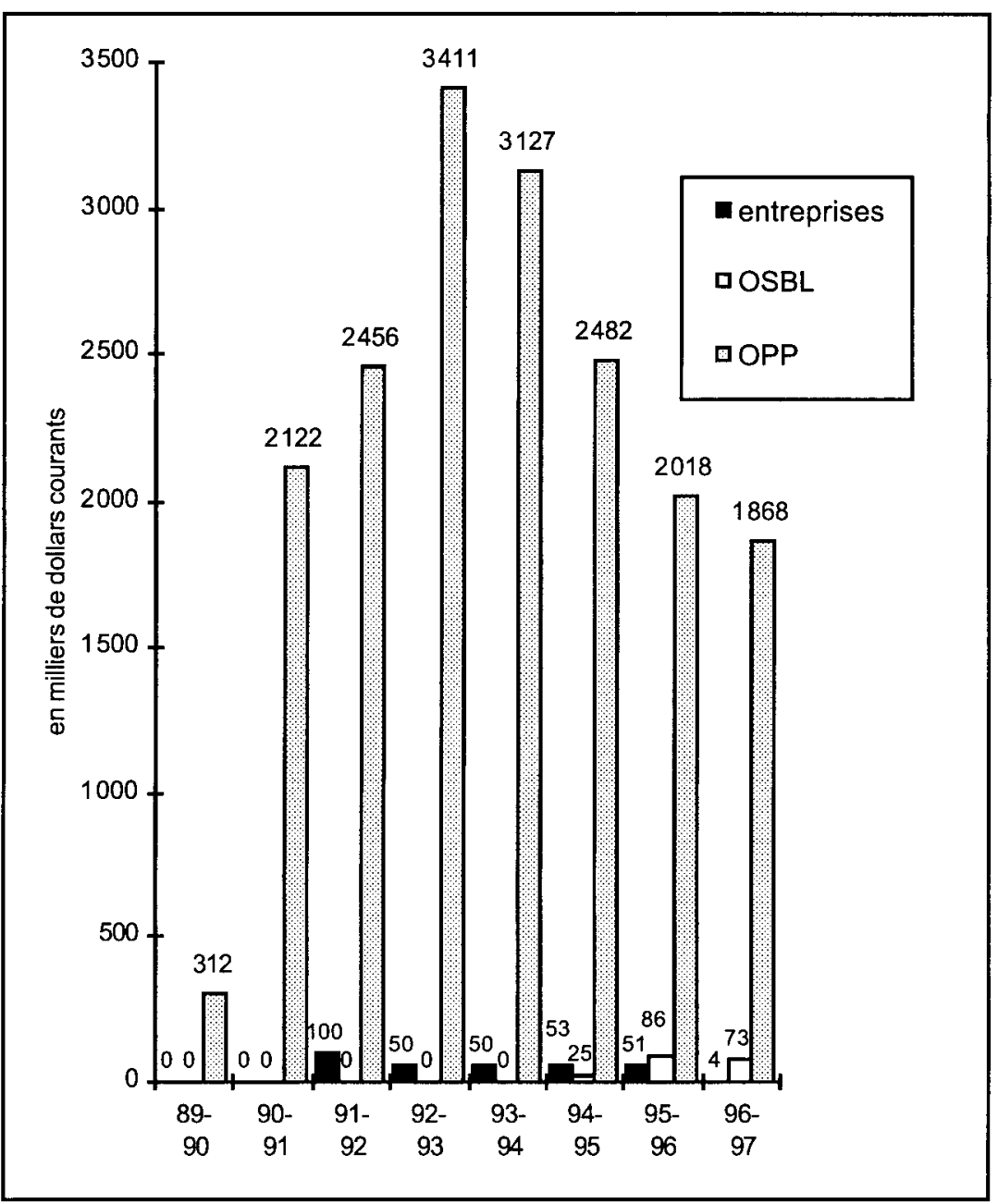

The Canadian Journal of Higher Education 
OPP forment la seule catégorie de partenaires à avoir contribué de façon ininterrompue au secteur des SHS au cours de la période ciblée, et qu'ils sont les seuls également dont l'apport annuel s'élève à plus d'un million de dollars. Si l'on fait exception de l'année 89-90, où leur appui n'a pas dépassé $312000 \$$ (en dollars courants), leur soutien aux SHS est en progression constante jusqu'en 92-93, culminant à 3, 4 millions $\$$, pour décliner par la suite et se fixer à 1, 9 millions $\$$ en $96-97$.

La première place occupée par les OPP au chapitre des investissements en SHS ne doit pas faire oublier toutefois que leur contribution y demeure inférieure à celle qu'ils versent au secteur des SNG (graphique 7). En effet, alors que la contribution des OPP aux SHS oscille entre 2 et 3 millions $\$$ (à l'exception de l'année $89-90$ où elle se chiffre à $312000 \$$ ), elle s'établit entre 5 et 9 millions $\$$ aux SNG (à l'exception de 1'année $89-90$ où elle se fixe à 3 millions $\$$ ). Il est plausible que cette différence puisse s'expliquer, comme nous l'avons mentionné plus tôt, par les coûts plus élevés de la recherche en SNG comparativement à ceux liés à la recherche en SHS. Cet écart en faveur des SNG remet toutefois en question l'idée généralement admise en sciences sociales, que les OPP, en raison de leurs besoins de connaissance en matières sociales, économiques, culturelles, etc. seraient portés à financer la recherche en SHS davantage qu'en SNG. Si cette idée s'était vérifiée, la part des fonds alloués aux SHS aurait dû s'établir à un niveau au moins équivalent à celui en SNG, ce que nos données infirment.

La contribution annuelle des OSBL et des entreprises aux SHS paraît, comparativement à celle des OPP, extrêmement modeste. Ce n'est pas avant 94-95 que les OSBL versent une première contribution, se chiffrant à $25000 \$$, aux SHS. Les investissements subséquents ne seront que très légèrement en hausse: $86000 \$$ en $95-96$ et $73000 \$$ en $96-97$. Pour ce qui est des entreprises, celles-ci accordent une contribution initiale de 100 $000 \$$ en $91-92$, puis fixent leur appui autour de $50000 \$$ pendant les quatre années suivantes, avant de la ramener à 4000 \$ en 96-97.

Dans le secteur des SNG (Graphique 7), les entreprises constituent la principale source de revenu additionnel pour les OS aux crédits gouvernementaux, surpassant de loin l'appui apporté par les OSBL et les OPP. En effet, alors que l'apport des entreprises s'élevait à 30 millions $\$$ en 
Graphique 7

Contribution des partenaires, par catégorie, aux programmes de partenariat en sciences naturelles et génie du Fonds FCAR et du CRSNG de 89-90 à 96-97

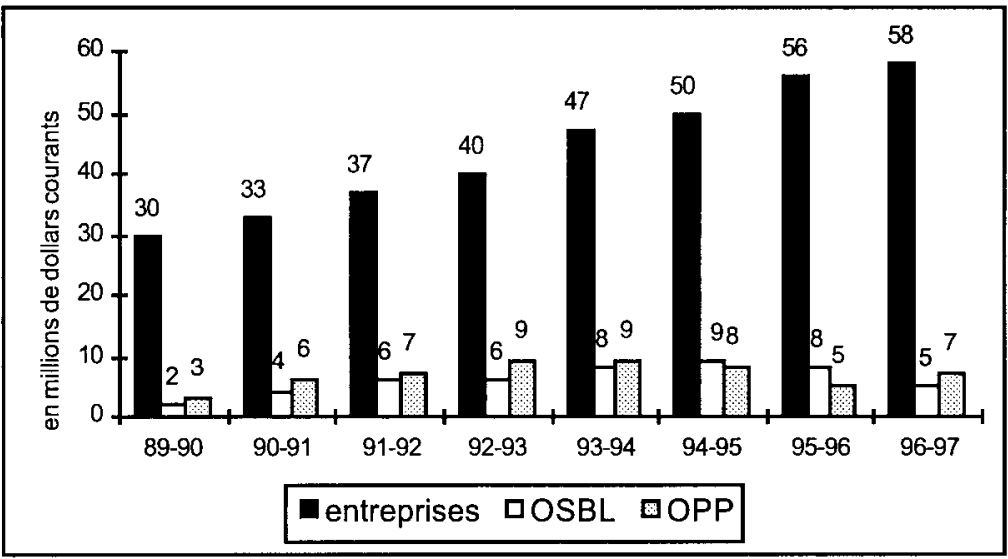

89-90, la contribution cumulée des OSBL et des OPP ne franchissait pas le cap des 5 millions \$. En 96-97, la contribution des entreprises se chiffrait à 58 millions $\$$ alors que celle des OSBL et des OPP se fixait à 12 millions \$.

Bien que la contribution des OSBL et des OPP affiche une croissance relative, en pourcentage, cinq fois plus élevée que celle des entreprises $(525 \%$ pour les OSBL et les OPP contre $90 \%$ pour les entreprises), la contribution des OSBL et des OPP demeure à ce point modeste qu'elle est incapable d'atteindre un niveau de financement proportionnellement comparable à celui des entreprises. Par exemple, pour les seules années 93-94 et 94-95, qui correspondent aux deux années où l'apport cumulé des OSBL et des OPP a été le plus substantiel (17 millions \$ par année), les entreprises versaient pour chacune de ces deux années 47 millions $\$$ et 50 millions $\$$, soit des contributions près de trois fois supérieures à celles des OSBL et des OPP réunis.

Ces données suggèrent que les tentatives des OS en SHS pour diversifier leurs sources de revenu ont donné lieu à un surinvestissement relatif de la part des OPP en comparaison avec les fonds versés par les 
OSBL et les entreprises. En fait, la diversification des sources de revenu souhaitée par les OS en SHS s'est traduite par une diversification des filières gouvernementales à travers lesquelles les fonds lui ont été acheminés plutôt qu'à un véritable élargissement des catégories de partenaires de la recherche. Les données du graphique 6 montrent bien, à cet égard, que les principaux partenaires dans les programmes de recherche orientée en SHS appartiennent dans une très large proportion à la catégorie des OPP, c'est-à-dire des institutions financées en tout ou en partie par des fonds publics (v.g. ministères, agences gouvernementales, instituts et centres de recherche publics).

Ces résultats convergent avec ceux obtenus par Godin (1998) dans une étude réalisée sur les pratiques de collaboration des chercheurs universitaires au Québec. Godin montre, en effet, que les activités de recherche menées en collaboration par les professeurs présentent des différences significatives selon le secteur disciplinaire auquel ils appartiennent. Les chercheurs rattachés aux secteurs des SNG et des SBM affichent un taux de collaboration avec l'industrie plus élevé que les chercheurs en SHS et en Arts et Lettres. Alors que 45\% des chercheurs en sciences naturelles, $47 \%$ en sciences biomédicales et $76 \%$ en génie collaborent avec l'industrie, ce taux ne s'établit qu'à $25 \%$ en Humanités, $18 \%$ en Arts et Lettres et $19 \%$ en sciences sociales. En ce qui a trait à la collaboration avec les organismes publics, les sciences sociales et les Humanités, avec un taux de collaboration respectif de $68 \%$ et de $64 \%$, devancent les sciences naturelles et les sciences biomédicales, mais sont à peu près à égalité avec le génie. Le taux de collaboration des chercheurs en génie avec le secteur public s'établit à $67 \%$, alors qu'il est de $55 \%$ en sciences naturelles et en sciences biomédicales.

Les chiffres rapportés par Godin (1998) appuient globalement les résultats de notre enquête et mettent en évidence la forte variation, selon les disciplines ou familles de disciplines, de l'intensité des liens entre le milieu universitaire et les institutions économiques et sociales. En se basant sur les liens de collaboration, Godin montre, en concordance avec nos résultats, la forte implication des OPP en génie. 


\section{La réponse des professeurs-chercheurs en sciences sociales aux programmes de subvention de recherche orientée}

En ce qui a trait à la réponse des professeurs-chercheurs dans le domaine des SHS à la mise sur pied et/ou à la consolidation des programmes de subvention de recherche orientée du CRSH, du CQRS et du Fonds FCAR (secteur SHS), les données exposées dans le graphique 8 suggèrent que ces derniers n'ont pas véritablement modifié leurs habitudes de recherche et ont continué à participer aux programmes de subvention de recherche non orientée davantage qu'à ceux de recherche orientée. La croissance de l'offre de subventions de recherche orientée semble ainsi ne pas avoir eu d'incidence apparente sur les activités de recherche des professeurs-chercheurs en SHS.

Le graphique 8 fait voir également que les variations annuelles enregistrées au chapitre des demandes de subventions en recherche orientée n'ont pas été accompagnées par une tendance à la hausse. En effet, après avoir enregistré plusieurs fluctuations au cours de la période ciblée, le nombre de demandes en 96-97 s'établit au même niveau qu'en 89-90, soit à 477 demandes. Les variations observées semblent principalement relever de facteurs conjoncturels, telle la création ou la transformation de programmes de subvention et l'établissement ou la cessation de partenariats entre les OS et les pourvoyeurs de fonds (l'établissement d'un partenariat se traduisant par le lancement d'un nouveau concours, sa cessation, par la suspension du concours, cf. Albert, 1999). Le nombre de demandes dans les programmes de recherche non orientée est lui aussi demeuré relativement stable. Cet état de fait peut probablement s'expliquer par la stagnation du nombre de postes de professeurs disponibles au Québec et au Canada au cours des années 90 .

Le graphique 9 indique, pour sa part, que le taux de demandes en recherche orientée par rapport au nombre de demandes en recherche non orientée se situe entre $20 \%$ et $33 \%$. Contrairement au nombre de demandes en recherche orientée (graphique 8) où les variations sont nombreuses, le taux de demandes en recherche orientée demeure relativement stable, autour de 23/24\% — à l'exception des années 93-94 
Graphique 8

Nombre de demandes de subventions dans les programmes de recherche orientée et non orientée en SHS au CRSH, au Fonds FCAR et au CQRS de 89-90 à 96-97

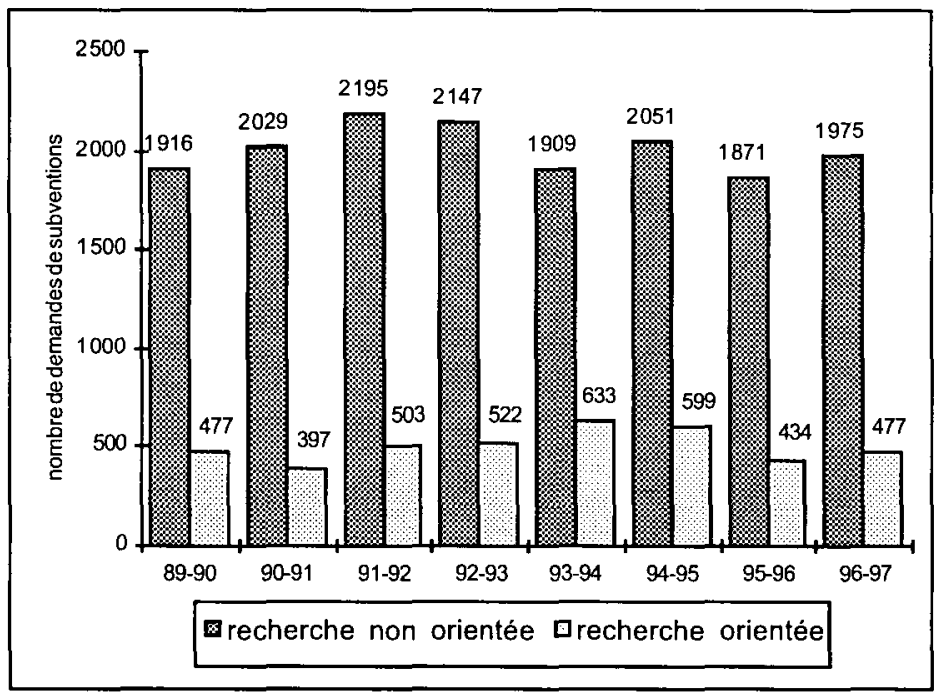

et $94-95$ où il s'établit à $33 \%$ et $29 \%$. Ces données permettent d'écarter l'hypothèse selon laquelle une tendance à la hausse en recherche orientée, qui n'aurait été décelable que par le biais de valeurs relatives, aurait pu échapper à notre attention. En concordance avec les données portant sur le nombre de demandes en recherche orientée, les données sur les taux de demandes tendent plutôt à confirmer le peu d'intérêt apparent manifesté par les professeurs en SHS à l'endroit des programmes de subvention de recherche orientée.

Contrairement à la thèse de Gibbons, Limoges, Nowotny, Schwartzman, Scott et Trow (1994), selon laquelle il y aurait eu une progression de la recherche à visée utilitaire ou en lien avec les contextes d'application, les données de notre enquête incite à penser que les professeurs-chercheurs en SHS n'ont pas modifié leurs activités au profit de la recherche axée sur la résolution de problèmes, ou l'étude de problèmes identifiés par les OS et les partenaires. Tel que l'indicateur du 
Graphique 9

Taux de demandes de subvention dans les programmes de subvention de recherche orientée en SHS sur le nombre de demandes dans les programmes de recherche non orientée au CRSH, au Fonds FCAR et au CQRS de 89-90 à 96-97

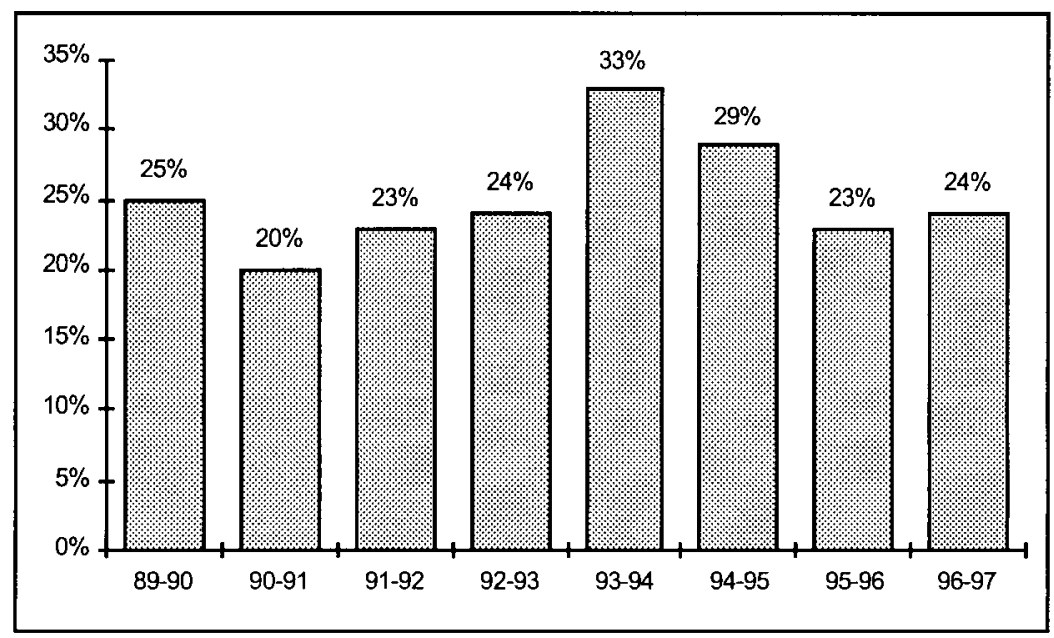

nombre de demandes de subvention permet de l'observer, on note plutôt une relative stabilité dans les pratiques de recherche des professeurs. Ces données sont congruentes avec deux études récentes portant sur les pratiques de recherche des professeurs en sociologie et en sciences économiques dans deux universités québécoises depuis les années 70 (Albert \& Bernard, 2000a, 2000b). Ces études suggèrent d'une part, que l'évolution des pratiques de recherche des professeurs n'est pas réductible à un modèle de compréhension linéaire et applicable à l'ensemble des disciplines, et d'autre part, qu'au delà des différences qui distinguent ces deux disciplines, les travaux destinés aux pairs majoritairement des travaux de recherche non orientée - ont acquis une valeur symbolique plus grande que les travaux visant la résolution de problème. Aucune de ces deux études n'a permis de vérifier l'hypothèse selon laquelle il y aurait eu une intensification significative de la recherche orientée ou à visée utilitaire. 


\section{CONCLUSION}

Cette étude visait à évaluer dans quelle mesure deux aspects de la stratégie déployée par les OS québécois et canadiens en SHS dans le but de préserver leur capacité de financement de la recherche universitaire dans le contexte du resserrement des finances publiques avaient atteint leurs objectifs. D'une part, il s'agissait de vérifier si la mise sur pied ou la consolidation de programmes de recherche orientée ont permis aux OS en SHS de générer des revenus additionnels à ceux octroyés par les gouvernements via les crédits parlementaires, d'autre part, vérifier dans quelle mesure les professeurs-chercheurs en SHS ont transformé leurs pratiques de recherche et répondu favorablement à l'accroissement de l'offre de subventions de recherche orientée. Au plan de l'analyse des dynamiques sociales, l'examen de ces deux aspects de la stratégie des OS permettait de documenter l'incidence de la dépendance accrue du mode d'allocation des ressources à l'endroit de la logique de marché sur la recherche universitaire.

En ce qui a trait à l'intensification du recours au partenariat comme mode de financement de la recherche, nos résultats ont fait ressortir que les SNG détenaient un pouvoir d'attraction auprès des pourvoyeurs de fonds nettement supérieur à celui des SHS. Ce pouvoir semblerait pouvoir s'expliquer d'une part, par leur capacité de générer des résultats susceptibles d'être convertis en innovations techniques ou en biens commercialisables, et d'autre part, en raison des mesures fiscales adoptées par les gouvernements en matière de R-D ayant pour effet de favoriser les SNG. Les SHS, en revanche, en raison du faible potentiel de commercialisation des résultats qu'elles génèrent et des contraintes entourant leur admissibilité aux régimes d'exemption fiscale, semblent ne pas bénéficier d'un potentiel équivalent. Nos résultats suggèrent ainsi que l'accentuation du recours au partenariat comme mode de financement de la recherche universitaire contribue à accentuer les inégalités économiques entre les SNG et les SHS.

Un raffinement accrue de nos catégories disciplinaires nous aurait probablement permis de faire ressortir des différences entre les disciplines elles-mêmes au sein de chacun des deux grands secteurs de 
connaissances que sont les SNG et les SHS. En effet, il est probable que des inégalités comparables à celles observées entre les SNG et les SHS soient apparues ou accentuées entre les disciplines elles-mêmes. Le niveau d'agrégation de nos données ne permettent pas toutefois de rendre compte de ces inégalités.

En ce qui concerne les mesures adoptées par les OS en SHS pour diversifier leurs sources de revenu, nos résultats ont montré que celles-ci ont donné lieu à une augmentation du nombre des OPP contribuant au financement de la recherche, mais non à un élargissement des catégories de partenaires. Les sommes additionnelles aux crédits parlementaires recueillis par les OS en SHS sont ainsi demeurées en tout ou en partie des fonds issus du secteur public; seules les filières gouvernementales à travers lesquelles celles-ci transitent ont été multipliées.

En ce qui a trait à la réponse des professeurs-chercheurs en SHS à la croissance de l'offre de subventions en recherche orientée, nos résultats suggèrent que ces derniers n'ont pas véritablement modifié leurs pratiques de recherche et ont continué à participer aux programmes de recherche non orientée davantage qu'aux programmes en recherche orientée. À la lumière de ce constat, la thèse de Gibbons et al. (1994), selon laquelle il y aurait eu une progression de la recherche en contexte d'application, ne semble pas se vérifier en SHS au Québec et au Canada.

\section{Les Notes}

1 Voir également CQRS, 1997, p. 10; CRSH, 1996, p. 2; Fonds FCAR, 1991, p. 1; 1993, p. 11; 1994, p. 9.

2 Voir également CRSNG, 1992, p. 2; 1994b, p. 2; 1995, p. 2; 1996, pp. 1-2; CRSH, 1989, p. 1; 1990b, pp. 1-2; 1994, p. 4, 8; CQRS, 1996, p. 14; University Affairs, 1996, p. 12.

3 Il importe de souligner toutefois qu'il peut exister une différence entre ce que les partenaires sont autorisés à faire et ce qu'ils font véritablement. En effet, il ne faut pas conclure que les bailleurs de fonds cherchent systématiquement à faire prédominer leurs intérêts parce qu'ils sont invités à participer au processus 
de sélection des projets. Il peut arriver que les partenaires préfèrent limiter leur participation à une évaluation superficielle de la pertinence socio-économique des projets soumis par les professeurs et n'avoir qu'une implication limitée dans l'orientation des travaux (Gingras, Godin \& Trépanier, 1999).

4 Pour une description détaillée des opérations méthodologiques liées à l'établissement des montants dépensés par année et par catégorie de partenaire, voir Albert, 1999.

5 Nous entendons par recherche orientée toute recherche sur laquelle les OS ou les partenaires exercent une influence directe sur le choix du sujet ou du thème de recherche, et par recherche non orientée toute recherche sur laquelle ni les OS ni les partenaires n'exercent une influence directe sur le choix du sujet ou thème de recherche.

6 Pour une description détaillée de tous les programmes de subvention de recherche orientée et non orientée examinées dans le cadre de l'étude, voir Albert, 1999, p. 241-245.

7 Un rapport du Conseil de la science et de la technologie du Québec faisait ressortir à cet égard que les contrats octroyés par les entreprises aux universités québécoises avaient connu une croissance de plus de $1200 \%$ entre 1984-1985 et 1995-1996, passant de 5,6 millions $\$$ à près de 76 millions $\$$ (1997, p. 35).

8 Le Bilan des activités du Plan de développement 1993-1996 du CQRS rapporte, à cet égard, qu'un projet d'action concertée entre le CQRS et une entreprise pharmaceutique aurait échoué en raison de l'absence de mesure d'exemption fiscale dans le secteur des SHS (CQRS, 1996, p. 24).

\section{Références}

Albert, M. (1999). Transformations des pratiques de recherche en sciences économiques et en sociologie dans deux universités québécoises: instrumentalisation de la production du savoir? Thèse de doctorat, Université de Montréal. www.pum.umontreal.ca/theses/catalogue.html

Albert, M., \& Bernard, P. (2000a). Faire utile ou faire savant? La «nouvelle production de connaissances» et la sociologie universitaire québécoise. Sociologie et sociétés, 32(1), 71-72. 
Albert, M., \& Bernard, M. (2000b). Sous l'empire de la science. La «nouvelle production de connaissances» et les sciences économiques universitaires québécoises. Sciences de la Société, 49, 27-46.

Beauchemin, J., Bourque, G., \& Duchastel, J. (1995). Du providentialisme au néolibéralisme: de Marsh à Axworthy. Un nouveau discours de légitimation de la régulation sociale. Cahiers de recherche sociologique, 24, 15-47.

Bourdieu, P. (1992). Les règles de l'art. Genèse et structure du champ littéraire. Paris: Seuil.

Bourdieu, P. (1991). The peculiar history of scientific reason. Sociological Forum, 6(1), 3-26.

Bourdieu, P. (1975). La spécificité du champ scientifique et les conditions sociale du progrès de la raison. Sociologie et sociétés, 7(1), 91-117.

Bourque, G., \& Beauchemin, J. (1994). La société à valeur ajoutée ou la religion pragmatique. Sociologie et sociétés, 26(2), 33-56.

Chapman, I. D., \& Farina, C. (1983). Peer review and national need. Research Policy, 12, 317-327.

Conseil de la science et de la technologie. (1997). Pour une politique québécoise de l'innovation. Québec.

CQRS. (1996). Bilan des activités du Plan de développement 1993-1996. Québec.

CQRS. (1997). Plan stratégique 1997-2000. Québec, QC.

CRSH. (1989). Nouvelles du CRSH, 2(2), 1. Ottawa, ON.

CRSH. (1990a). Regard sur l'avenir. Ottawa, ON.

CRSH. (1990b). Nouvelles du CRSH, 3(1), 1-2. Ottawa, ON.

CRSH. (1994). Nouvelles du CRSH, 7(2), 4-8. Ottawa, ON.

CRSH. (1996). Le défi du juste milieu, Plan quinquennal 1996-2001. Ottawa, ON.

CRSNG. (1992). Contact, 17(3), 2. Ottawa, ON.

CRSNG. (1994a). Contact, 19(2), 1-2. Ottawa, ON.

CRSNG. (1994b). Contact, numéro spécial, 2-3. Ottawa, ON.

CRSNG. (1995). Contact, 20(2), 2. Ottawa, ON.

CRSNG. (1996). Contact, 21(2), 1-2. Ottawa, ON.

CRSNG. (1999). http://www.nserc./ca/about/stats/francais/table_21f.htm.

Fonds FCAR. (1991). Objectif Recherche, 3(1), 1. Québec, QC.

Fonds FCAR. (1993). Rapport annuel 1992-1993. Québec, QC.

Fonds FCAR. (1994). Rapport annuel 1993-1994. Québec, QC.

Fonds FCAR. (1996). Portrait de la clientèle du Fonds FCAR: 1996-1997, Évaluation interne 1995-1996: partie 2. Québec, QC. 
Davies, C. H. (1993). La politique technologique du gouvernement du Québec. In R. Dalpé \& R. Landry (Eds.), La politique technologique au Québec (pp. 99-128). Montréal, QC: Presses de l’Université de Montréal.

Dalpé, R. (1999, mai). Le financement public de la recherche universitaire. Le public et le privé en science. Université, 16-18.

Dalpé, R., \& Gingras, Y. (1990). Recherche universitaire et priorités nationales: l'effet du financement public sur la recherche en énergie solaire au Canada. La revue canadienne d'enseignement supérieur, 20(2), 27-44.

Dalpé, R., \& Anderson, F. (1995). National priorities in academic research, strategic research and contracts in renewable energies. Research Policy, 24, $563-581$.

Dufour, P., \& Gingras, Y. (1993). La politique scientifique et technologique du gouverne-ment du Canada. In R. Dalpé \& R. Landry (Eds.), La politique technologique au Québec (pp. 129-141). Montréal, QC: Presses de l'Université de Montréal.

Gibbons, M., Limoges, C., Nowotny, H., Schwartzman, S., Scott, P., \& Trow, M. (1994). The new production of knowledge. The dynamics of science and research in contemporary societies. London, Thousand Oaks, New Delhi: Sage Publications.

Gingras, Y., Godin, B., \& Trépanier, M. (1999). La place des universités dans les politiques scientifiques et technologiques canadiennes et québécoises. In P. Beaulieu \& D. Bertrand (Eds.), L'État québécois et les universités: acteurs et enjeux (pp. 69-99). Sainte-Foy, QC: Presses de l'Université du Québec.

Godin, B. (1998). Writing Performative History: The New New Atlantis? Social Studies of Science, 28(3), 465-483.

Godin, B., \& Landry, R. (1995). L'avenir de la collaboration scientifique au Québec: une analyse basée sur la convergence d'indicateurs. Rapport présenté au Fonds FCAR. Québec.

Godin, B., \& Trépanier, M. (1995). La politique scientifique et technologique québécoise: la mise en place d'un nouveau système national d'innovation. Recherches sociographiques, 36(3), 445-477.

Leclerc, M. (1991). Les relations université-entreprise entre l'État et le besoin. La revue canadienne d'enseignement supérieur, 21(1), 54-70.

Leclerc, M., \& Gingras, Y. (1993). Les indicateurs du financement privé de la R-D universitaire au Québec: critique de la méthode. La revue canadienne d'enseignement supérieur, 23(1), 74-107. 
Limoges, C. (1990). De la politique des sciences à la politique de l'innovation: l'État incertain. In M. Leclerc (Ed.), Les enjeux économiques et politiques de l'innovation (pp. 61-79). Sillery, QC: Presses de l'Université du Québec.

Pollack, M. (1975). L'efficacité par l'ambiguité. La transformation du champ scientifique par la politique scientifique: le cas de la sociologie et des sciences économiques en France. Sociologie et sociétés, 7(1), 29-49.

Slaughter, S., \& Leslie, L. L. (1997). Academic capitalism. Politics, policies and the entrepreurial university. Baltimore, London: Johns Hopkins University Press.

SSHRC. (March 1989). Report of the Second Task Force on Priorities. Ottawa, ON.

SSHRC. (April 1996). Strategic Grant Programs Review, Final Report. Ottawa, ON.

Trépanier, M. (1992). Politique de la science au Québec et autonomie du champ scientifique. Revue québécoise de science politique, (22), 101-135.

University Affairs (March 1996). pp. 12-13. 